\title{
Establishing a Comprehensive Ethics-Based Checklist for Surgical Camp Activities at AMPATH-Kenya
}

\author{
Diane B. Choi ${ }^{1}$, BS, Kyle L. Carpenter ${ }^{2}$, MD, Connie H. Keung ${ }^{2,3}$, MD
}

${ }^{1}$ Indiana University School of Medicine, ${ }^{2}$ Indiana University School of Medicine, Department of Surgery, ${ }^{3}$ Moi Teaching and Referral Hospital, Eldoret, Kenya

Background and Hypothesis: Global health has made strides in controlling and reducing harm from infectious diseases such as Polio and HIVIAIDS but this focus has not been mirrored in surgical and anesthesia care, especially in many low and middle-income countries (LMICs). In 2010, an estimated 16.9 million lives were lost from conditions that required surgical care and 5 billion people in LMICs lack safe and affordable surgical and anesthesia. Organization of surgical camps can bring skilled physicians to temporarily meet the unmet surgical needs in underserved areas. However, currently there are no government agencies or accrediting bodies existing to assess the scope/impact of short-term surgical camp care or establish standards of care for such services.

Experimental Design or Project Methods: The AMPATH mission of "start with healthcare and then do more to empower communities, educate tomorrow's medical experts and research breakthroughs that will truly change the health of the world" was incorporated into the ethical checklist proposed. The four principles beneficence, non-maleficence, autonomy, and justice were used as guidance for the checklist.

Results: The checklist can be organized into three main areas: Care, Empowerment \& Training, and Research (Figure 1).

\section{广. ampath}

Surgical Camp Checklist

Care/Treatment

A. Pre-Operative Care and Preparation

1. Pre-Departure Cultural Competency

2. Needs Assessment

3. Clinic and Patient Evaluation

4. Multidisciplinary Case Conference

5. Appropriate Documentation and Preparation

B. Practices in the Operating Room and During Surgery (Intraoperative Care)

1. Maintaining a Standard of Care

2. Avoidance of Unnecessary Risk

3. Prevention of Disruption of Care

4. Appropriate Staffing

5. Mutual Learning

C. Post-Operative Care

1. Patient Follow-Up

2. Rounding on Patients Post-Operatively

3. Post-Departure Debriefing

4. Morbidity and Mortality Conference

Empowerment \& Training

1. Investment in Personnel

2. Mutual Learning

3. Empowerment through Discussion

Research

1. Data Collection

2. Evaluation of Programs to Measure Impact and Quality

Figure 1: Surgical Camp Checklist to be implemented by all surgical camps working with AMPATH-Surgery.

Conclusion and Potential Impact: By structuring the surgical camp ethical guidelines in a way that first meets a need (pre-operative evaluation and surgery), links people with care (follow-up) and then goes beyond care (research and education), AMPATH Surgery can continue to build upon the already existing relationships with stakeholders while improving outcomes and population health. The authors' hope that similar ethical guidelines be enforced in future surgical camp activities in other organizations. 